\title{
Dickkopf-3 maintains the PANC-1 human pancreatic tumor cells in a dedifferentiated state
}

\author{
CHRISTOPH ZENZMAIER ${ }^{1}$, MARTIN HERMANN ${ }^{2}$, PAUL HENGSTER ${ }^{2}$ and PETER BERGER ${ }^{1}$ \\ ${ }^{1}$ Institute for Biomedical Aging Research, Austrian Academy of Sciences, Rennweg 10; \\ ${ }^{2}$ KMT Laboratory, Department of Visceral-, Transplant- and Thoracic Surgery, Center of Operative \\ Medicine, Innsbruck Medical University, Innrain 66, A-6020 Innsbruck, Austria
}

Received June 22, 2011; Accepted July 15, 2011

DOI: $10.3892 /$ ijo.2011.1180

\begin{abstract}
Pancreatic cancer $(\mathrm{PaCa})$ is the fourth leading cause of cancer deaths in Western societies, with pancreatic ductal adenocarcinomas (PDACs) accounting for $>90 \%$ of such cases. PDAC is a heterogeneous disease that includes a subset showing overexpression of the secreted glycoprotein Dickkopfrelated protein 3 (Dkk-3), a protein shown to be downregulated in various cancers of different tissues. The biological function of Dkk-3 in this subset was studied using the Dkk-3 expressing PANC-1 cell line as a model for PDACs. The influence of Dkk-3 overexpression and knockdown on cellular differentiation and proliferation of PANC-1 was investigated. Confocal microscopy showed that Dkk-3 was expressed in a fraction of PANC-1 cells. While lentiviral-mediated overexpression of $D K K 3$ did not alter cellular proliferation, knockdown of $D K K 3$ resulted in significant reduction of cellular proliferation and concomitant induction of cell cycle inhibitors $C D K N 2 B\left(\mathrm{p} 15^{\mathrm{INK} 4 \mathrm{~b}}\right), C D K N 1 A\left(\mathrm{p} 21^{\mathrm{CIP} 1}\right)$ and $C D K N 1 B\left(\mathrm{p} 27^{\mathrm{KIP} 1}\right)$. In parallel, pancreatic epithelial cell differentiation markers $A M Y 2 A, C E L A 1, C T R B 1, G C G, G L B 1$ and INS were significantly upregulated. PANC-1 cells differentiated using exendin-4
\end{abstract}

Correspondence to: Dr Peter Berger, Institute for Biomedical Aging Research, Austrian Academy of Sciences, Rennweg 10, A-6020 Innsbruck, Austria

E-mail: peter.berger@oeaw.ac.at

Abbreviations: AMY2A, amylase $\alpha 2 \mathrm{~A}$; CELA1, chymotrypsin-like elastase family member 1; CTRB1, chymotrypsinogen B1; Dkk-3, Dickkopf-3; GCG, glucagon; GLB1, galactosidase $\beta 1$; IEMA, immunoenzymometric assay; INS, insulin; KD DKK3, lentiviraldelivered shRNA targeting $D K K 3$; KD SCR, lentiviral-delivered shRNA targeting scrambled sequence; OE DKK3, lentiviral overexpression of $D K K 3$; OE VEC, lentiviral overexpression of empty control vector; $\mathrm{PaCa}$, pancreatic cancer; PDAC, pancreatic ductal adenocarcinoma; qPCR, quantitative real-time PCR; SA- $\beta-G a l$, senescence-associated $\beta$-galactosidase

Key words: Dickkopf-3, differentiation, pancreatic cancer, pancreatic ductal adenocarcinoma, proliferation, senescence-associated $\beta$-galactosidase showed analogous induction of cell cycle inhibitors and differentiation markers. Thus, we conclude that Dkk-3 is required to maintain a highly dedifferentiated and consequently proliferative state in PANC-1, indicating a similar function in the Dkk-3 overexpressing subset of PDACs. Therefore, Dkk-3 represents a potential target for the treatment of Dkk-3-positive subtypes of $\mathrm{PaCa}$ to drive cells into cell cycle arrest and differentiation.

\section{Introduction}

Pancreatic cancer $(\mathrm{PaCa})$ is the fourth leading cause of cancer deaths in Western societies. In 2008, there were an estimated 68,500 new cases and 70,200 deaths from the disease in the European Union (1), indicating incidence rates approximately equal to mortality. Clearly $\mathrm{PaCa}$ has an extremely poor prognosis, with a five-year relative survival rate of only $5 \%$ (2). Because PaCa-specific symptoms occur late in the course of the disease, for the majority of patients curative resections are not usually possible. Median survival from time of diagnosis is 3.5 months for patients who were not resected and 13.3 months for those who underwent resection (3). Thus, novel targets for early detection and therapy of PaCas are urgently needed. A better understanding of the underlying molecular and cellular changes associated with dedifferentiation and proliferation of pancreatic tumor cells is a prerequisite to achieve this purpose.

The secreted glycoprotein Dickkopf-related protein 3 (Dkk-3) is the most divergent member of the human Dickkopf family $(4,5)$ and, in contrast to other family members, does not modulate Wnt signaling (6,7). Dkk-3 has been proposed to represent a novel tumor suppressor since gene expression is downregulated in various tumor cells (8-12), and hypermethylation of its promoter correlates with cancer occurrence $(13,14)$. However, overexpression of Dkk-3 does not influence proliferation of malignant and non-malignant prostate cells (15).

In a cancer-profiling array, Dkk-3 was downregulated in 5 of $7 \mathrm{PaCa}$ samples, but was upregulated in the remaining two (8). Additionally, Dkk-3 expression in tumor epithelial cells was demonstrated in $\sim 17 \%$ of pancreatic ductal adenocarcinomas (PDACs) by immunohistochemistry (16), while exocrine or ductal cells of normal adult pancreatic tissue were negative (17), indicating a role for Dkk-3 in a subset of PDACs. Malignant neoplasms arise mainly from exocrine regions of the gland with PDACs accounting for $>90 \%$ of $\mathrm{PaCa}$ cases. 
In the present study, the role of Dkk-3 was investigated in the PANC-1 cell line, an extensively used model for human PDAC (18-23). PANC-1 cells have previously been shown to express $D K K 3$ at mRNA levels (24). Herein, we demonstrate that Dkk-3 supports proliferation of PANC-1 cells as shown by knockdown of Dkk-3 via lentiviral-delivered shRNA. Dkk-3 knockdown induced the expression of several markers of the differentiated pancreas, indicating that Dkk-3 plays a role in maintaining a dedifferentiated state in the cell line PANC-1 as a prerequisite for proliferation.

\section{Materials and methods}

Cell culture and cellular differentiation. PANC-1 cells were purchased from the American Type Culture Collection (ATCC) and were culture in DMEM containing $4.5 \mathrm{~g} / 1$ glucose (Lonza), $10 \%$ bovine calf serum (BCS, HyClone) and $1 \%$ penicillin/ streptomycin/L-glutamine (PAA Laboratories). To induce PANC-1 differentiation, cells were seeded in 6-well plates in DMEM/F12 (Sigma-Aldrich) containing 1\% BSA and ITS. At day 4 of culture, fresh media were supplemented with $0.3 \mathrm{mM}$ taurine (Sigma-Aldrich), and at day 10, media were replaced by fresh media containing 1.5\% BSA, ITS (Sigma-Aldrich), $3 \mathrm{mM}$ taurine (Sigma-Aldrich), $1 \mathrm{mM}$ nicotinamide (SigmaAldrich), NEAA (BD Biosciences) and $100 \mu \mathrm{M}$ exendin-4 (Sigma-Aldrich). Media were replaced every other day. Cells were harvested at day 17. Control cells were maintained in DMEM containing $10 \%$ BCS.

Immunofluorescence and confocal microscopy. Cell growth and live confocal imaging was performed in 8 well-chambered coverglasses (Nalge Nunc International). After fixation, the cells were washed in phosphate-buffered saline solution (PBS, three times at RT) and non-specific binding was blocked with $3 \%$ BSA/PBS for one hour at room temperature. The primary antibody was goat anti-human Dkk-3 ( $1 \mu \mathrm{g} / \mathrm{ml}$, R\&D Systems) diluted in $1 \% \mathrm{BSA} / \mathrm{PBS}$, incubated for $1 \mathrm{~h}$ at $37^{\circ} \mathrm{C}$ in a humidified chamber. The cells were washed and blocked again as described previously. The secondary antibody (rabbit anti-goat rhodamine, $10 \mu \mathrm{g} / \mathrm{ml}$; Chemicon) was incubated for $1 \mathrm{~h}$ at $37^{\circ} \mathrm{C}$. After the final two washing steps, nuclei were stained with Sytox green nucleic acid stain (Molecular Probes-Invitrogen Life Technologies) $10 \mathrm{nM}$ in PBS, and incubated for $20 \mathrm{~min}$ at $37^{\circ} \mathrm{C}$.

Confocal images were captured with an UltraVIEW RS (Perkin-Elmer) mounted on an Olympus IX-70 inverse microscope (Olympus) using a 40x/1.4 oil immersion objective. The results presented are from representative fields. Live confocal microscopy was performed with a microlens-enhanced Nipkow disk-based confocal system UltraVIEW RS (Perkin-Elmer) in combination with the live stains fluorescent wheat germ agglutinin (WGA; Molecular Probes), tetramethylrhodamine methyl ester perchlorate (TMRM; Sigma-Aldrich) and Rhod-2 (Molecular Probes).

Knockdown and overexpression of DKK3 by lentiviral particles. Production of lentiviral particles was carried out according to the manufacturer's protocol (Addgene). For knockdown of $D K K 3$, the lentiviral pLKO.1-TRC short hairpin system (Addgene) was applied. The target sequence (5-gagcctgatggag cettggaccgat) was located within the coding region of $D K K 3$. In knockdown experiments, scramble shRNA vector (Addgene plasmid 1864) was used as control. For overexpression, fulllength cDNA of $D K K 3$ was subcloned into the pLenti6 vector (Invitrogen). Empty vector was used as control. Virus particles were produced by cotransfection of HEK293FT cells (Invitrogen) with the lentivirus packaging plasmid (psPAX2, Addgene plasmid 12260), envelope coding plasmid (pMD2.G, Addgene plasmid 12259) and vector construct. The lentivirus-containing supernatants were aliquoted and stored at $-80^{\circ} \mathrm{C}$. For viral transduction, cells were seeded in appropriate vessels and left to adhere overnight. Thereafter, medium was replenished and supplemented with virus-containing supernatant at MOI 4 (knockdown) and MOI 0.5 (overexpression), respectively.

Quantitative real-time PCR. mRNA was extracted by the TriFast $^{\mathrm{TM}}$ reagent (PeQLAB Biotechology). cDNA first strand synthesis was reverse transcribed from $2 \mu \mathrm{g}$ total RNA preparation using Reverse Transcription System (Promega) and oligo(dT)15 and random hexamer primers. qPCR was performed by the FastStart DNA Master SYBR-Green I kit and the Light Cycler 480 System (Roche Applied Science) according to manufacturer's instructions. Specificity of PCR products was confirmed by melting curve analysis. Primer sequences are given in Table I. cDNA concentrations were normalized by the housekeeping gene porphobilinogen deaminase $(H M B S)$.

Dkk-3 quantification in cell culture supernatants by IEMA. PANC-1 cells were seeded at a density of $1 \times 10^{5} /$ well in 6 -well plates and left to adhere overnight. Subsequently, medium was replaced and cells were transduced with lentiviral particles. After $72 \mathrm{~h}$, medium was replaced by $1 \mathrm{ml}$ of fresh medium. Levels of Dkk-3 secreted into the medium within $24 \mathrm{~h}$ were quantified by a sandwich immunoenzymometric assay (IEMA) based on our own monoclonal antibody, as previously described $(25,26)$.

Cell viability assay. Two thousand cells were seeded in triplicates into 96-well plates (Nunc) in $100 \mu \mathrm{l}$ culture medium and left to adhere overnight. Thereafter, fresh medium was supplemented with lentivirus particles to transduce cells. Cell viability was determined by a WST-1 cell viability assay (Roche Applied Science) according to the manufacturer's instructions at 24, 72 and $144 \mathrm{~h}$ post-transduction (days 1, 3 and 6, respectively). Media were replaced after $48 \mathrm{~h}$.

Relative quantification of DNA synthesis. Two thousand cells were seeded in triplicates into 96-well plates (Nunc) in $100 \mu \mathrm{l}$ culture medium and left to adhere overnight. Thereafter, fresh medium was supplemented with lentivirus particles to transduce cells. Proliferation rate was analyzed by a BrdU cell proliferation ELISA (Roche Applied Science) according to manufacturer's instructions at 24,72 and $144 \mathrm{~h}$ post-transduction (days 1, 3 and 6, respectively). Media were replaced after $48 \mathrm{~h}$.

Western blot analysis. Total cell extracts were prepared and analyzed by Western blotting as described previously (15). Primary antibodies against PARP $\alpha$, phospho-p53, p15 ${ }^{\text {INK4b }}$, p21 ${ }^{\mathrm{CIP} 1}, \mathrm{p} 27^{\mathrm{KIP} 1}$ (Cell Signaling Technology) and Dkk-3 (R\&D 

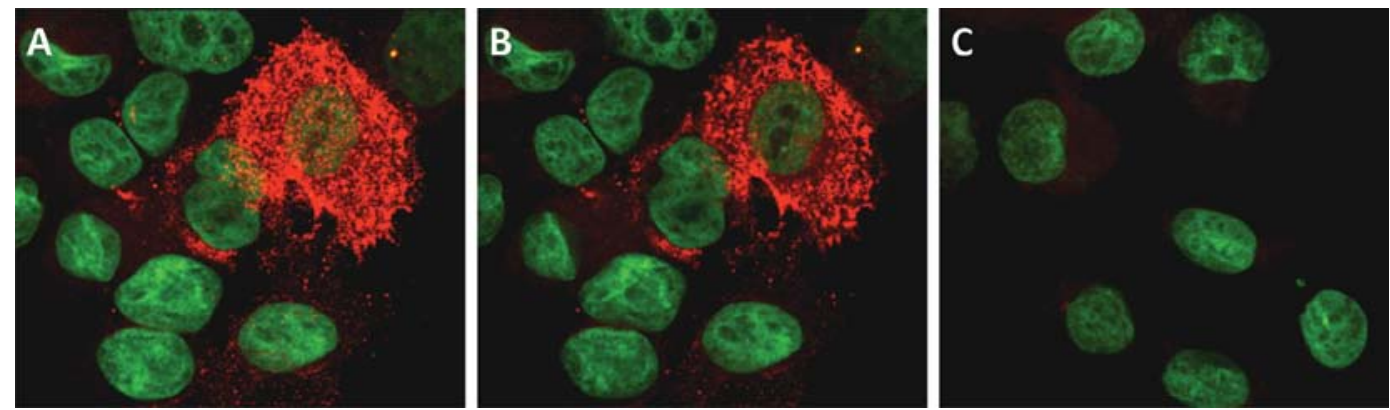

Figure 1. Dkk-3 expression in PANC-1 cells. Confocal immunofluorescence of PANC-1 cells stained with an antibody directed against Dkk-3 (red). Cell nuclei were counterstained with Sytox (green). (A) Confocal z-stack of PANC-1 cells reveal heterogeneous expression of Dkk-3. (B) The staining reaction for Dkk-3 in PANC-1 cells in one confocal plane was distributed in a vesicular pattern in the cytoplasm, while the cell nuclei were not stained. (C) Specificity of the staining was demonstrated by omitting the primary antibody (z-stack).

Table I. Primer sequences.

Primer sequences

\begin{tabular}{llll} 
Gene & Unigene ID & \multicolumn{1}{c}{ Sense } & \multicolumn{1}{c}{ Antisense } \\
\hline$A M Y 2 A$ & Hs.654437 & 5-atgtggcctggagacataaa & 5-ccattccacttgcgaataac \\
$B A K 1$ & Hs.485139 & 5-tctggcctacacgtctacc & 5-acaaactggcccaacagaac \\
$B A X$ & Hs.433670 & 5-tttgcttcagggttcatcc & 5-cagttgaagttgccgtcaga \\
$B C L 2$ & Hs.150749 & 5-gaggattgtggccttctttg & 5-acagttccacaaaggcatcc \\
$B C L 2 L 1$ & Hs.516966 & 5-catggcagcagtaaagcaag & 5-tgctgcattgttcccataga \\
$C D K N 1 A\left(\mathrm{p} 21^{\mathrm{CIP1}}\right)$ & Hs.370771 & 5-ggcggcagaccagcatgacagatt & 5-gcagggggcggccagggtat \\
$C D K N 1 B\left(\mathrm{p} 27^{\mathrm{KIP1}}\right)$ & Hs.238990 & 5-aataaggaagcgacctgcaa & 5-cgagctgtttacgtttgacg \\
$C D K N 2 B\left(\mathrm{p} 15^{\mathrm{INK} 4 \mathrm{~b}}\right)$ & Hs.72901 & 5-cggggactagtggagaaggt & 5-ggtgagagtggcagggtct \\
$C E L A 1$ & Hs.348395 & 5-ggagtgaccagctttgtgtc & 5-aaggtcgttggactcaggaa \\
$C T R B 1$ & Hs.610926 & 5-accgacgtgatgatctgtg & 5-gatcttctgcacccaaggta \\
$D K K 3$ & Hs.292156 & 5-tcatcacctgggagctagag & 5-caacttcatactcatcgggg \\
$G C G$ & Hs.516494 & 5-cgttccttcaagacacaga & 5-acgcctggagtccagatact \\
$G L B 1$ & Hs.443031 & 5-tcctccatctacaccaaag & 5-tgaaggctactgttcac \\
$H M B S$ & Hs.82609 & 5-ccaggacatcttggatctgg & 5-atggtagcctgcatggtctc \\
$I N S$ & Hs.272259 & 5-acgaggcttcttctacacacc & 5-agctggtagagggagcagat \\
$T P 53(\mathrm{p} 53)$ & Hs.654481 & 5-gcgcacagaggaagagaa & 5-cctcattcagctctcggaac \\
\hline
\end{tabular}

Systems) were used at dilutions of 1:1000, against BAX (Oncogene) at a dilution of 1:500, and against $\beta$-actin (SigmaAldrich) at a dilution of 1:5000, respectively.

Staining of senescence-associated $\beta$-galactosidase activity. For senescence-associated $\beta$-galactosidase (SA- $\beta$-Gal) activity staining, cells were fixed ( $2 \%$ formaldehyde, $0.2 \%$ glutaraldehyde in PBS) for $5 \mathrm{~min}$ at room temperature and subsequently incubated in staining solution $[40 \mathrm{mM}$ citric acid, $12 \mathrm{mM}$ $\mathrm{Na}_{2} \mathrm{HPO}_{4}, 150 \mathrm{mM} \mathrm{NaCl}, 2 \mathrm{mM} \mathrm{MgCl}{ }_{2}, 5 \mathrm{mM} \mathrm{K}_{3} \mathrm{Fe}(\mathrm{CN})_{6}$, $5 \mathrm{mM} \mathrm{K}_{4} \mathrm{Fe}(\mathrm{CN})_{6}, 1 \mathrm{mg} / \mathrm{ml}$ 5-bromo-4-chloro-3-indolyl- $\beta$ D-galactoside (X-Gal), $\mathrm{pH} \mathrm{6.0]} \mathrm{for} 24 \mathrm{~h}$ at $37^{\circ} \mathrm{C}$. Cells were washed in PBS to stop the reaction.

Statistics. Results are expressed as the mean values \pm SEM. Statistical differences between treatments were calculated by paired Student's t-test and regarded significant when $\mathrm{P}<0.05$ $\left({ }^{*} \mathrm{P}<0.05,{ }^{* *} \mathrm{P}<0.01\right)$.

\section{Results}

Dkk-3 is heterogeneously expressed in PANC-1 cells. To investigate cellular distribution of Dkk-3 protein in PANC-1 cells, confocal immunofluorescence using Sytox green as a counter stain for cell nuclei was performed. PANC-1 cells stained heterogeneously positive for Dkk-3 (Fig. 1A). While the majority of cells had no or only weakly detectable Dkk-3 expression, approximately $10 \%$ of the cells were intensely stained. As expected for a secreted protein, Dkk-3 was distributed in a vesicular pattern within the cytoplasm while cell nuclei were negative (Fig. 1B).

DKK3 knockdown reduces proliferation of PANC-1. To investigate the role of Dkk-3 in PANC-1 cells, lentiviral $D K K 3$ overexpression and knockdown systems were established. The efficiency of the transduction system was confirmed at mRNA and protein levels using quantitative real-time PCR (qPCR) 
and IEMA, respectively (Fig. 2A). Lentiviral overexpression of $D K K 3$ (OE DKK3) increased mRNA levels 586-fold $(\mathrm{n}=4$, $\mathrm{P}<0.001)$ compared with empty vector control virus (OE VEC) in PANC-1 cells. The amount of secreted Dkk-3 consistently increased from 18.1 to $5073 \mathrm{ng} / \mathrm{ml}$ (280-fold, $\mathrm{n}=3, \mathrm{P}=0.03$ ). Lentiviral-delivered shRNA targeting $D K K 3$ (KD DKK3) led to an $88 \%$ reduction (8.4-fold decrease, $n=3, P=0.004)$ of DKK3 mRNA level compared with scrambled control (KD $\mathrm{SCR}$ ), and a $98 \%$ reduction (50-fold decrease, $\mathrm{n}=3, \mathrm{P}=0.01)$ of secreted Dkk-3 protein. Neither cell morphology (visualized with WGA), mitochondrial activity (visualized with TMRM) nor calcium levels (visualized with Rhod-2) showed clear differences between $D K K 3$ overexpressing and knockdown cells compared to controls (data not shown).

Given the proposed function of Dkk-3 as a tumor suppressor, the effect of $D K K 3$ overexpression on proliferation of PANC-1 cells was investigated by the established lentiviral transduction system. In agreement with our previous findings in malignant and nonmalignant prostate cells (15), OE DKK3 did not significantly alter cellular proliferation at any time point tested (days 1,3 and 6 post-transduction) as determined by cell viability (WST-1) and BrdU incorporation (DNA synthesis) assays (Fig. 2B). KD DKK3 did not significantly alter proliferation of PANC-1 cells within 1 or 3 days post-transduction. However, after 6 days, KD DKK3 PANC-1 showed significantly reduced proliferation compared with KD SCR (Fig. 2C; WST-1 signal

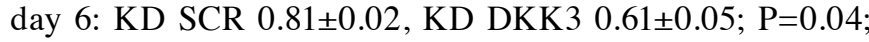
BrdU signal day 6: KD SCR 1.92 \pm 0.07 , KD DKK3 1.17 \pm 0.18 ; $\mathrm{P}=0.02$ ). Of note, KD DKK3 did not attenuate proliferation of Dkk-3 negative cell lines (PC3 prostate cancer cell line, HT-29 colon carcinoma cell line) excluding potential Dkk-3 independent effects of the lentiviral knockdown system (data not shown).

Elevated p27 levels in DKK3 knockdown cells. To gain insight into the molecular mechanisms underlying the reduced proliferation of PANC-1 upon KD DKK3, expression levels of several genes related to apoptosis were analyzed. KD DKK3 PANC-1 revealed no significant changes in expression levels of BAK1, BCL2L1 and TP53 (p53) compared with KD SCR PANC-1 (Fig. 3A). While mRNA levels of the anti-apoptotic BCL2 was slightly, but not statistically significantly, elevated (3.1-fold, $\mathrm{P}=0.06$ ), the pro-apoptotic $B A X$ was significantly downregulated (-1.5-fold, $\mathrm{P}=0.013)$. However, at protein level, neither BAX nor the apoptosis markers PARP $\alpha$ and phosphop53 were regulated, indicating that KD DKK3 did not induce apoptosis in PANC-1 cells (Fig. 3B).

Additionally, expression levels of several cell cycle-dependent kinase inhibitors were analyzed. $C D K N 2 B\left(\mathrm{p} 15^{\mathrm{INK} 4 \mathrm{~b}}\right.$; 8.1-fold, $\mathrm{P}=0.03), C D K N 1 A$ (p21 ${ }^{\mathrm{CIP} 1} ; 2.9$-fold, $\left.\mathrm{P}=0.04\right)$ and $C D K N 1 B$ (p27 ${ }^{\mathrm{KIP} 1} ; 3.4$-fold, $\mathrm{P}=0.003$ ) were significantly induced in KD DKK3 compared with KD SCR PANC-1 (Fig. 3C). The induction of $\mathrm{p} 27^{\mathrm{KIP} 1}$ was confirmed at the protein level by Western blot analysis, while protein levels of $\mathrm{p} 15^{\mathrm{INK} 4 \mathrm{~b}}$ and p $21^{\mathrm{CIP} 1}$ were not altered (Fig. 3D).

DKK3 knockdown induces differentiation of PANC-1 cells. $\mathrm{p} 27^{\mathrm{KIP} 1}$ plays an important role in the switch of proliferation to differentiation in a variety of cell types, and loss of $\mathrm{p} 27^{\mathrm{KIP} 1}$ is associated with a poorly differentiated phenotype in several
A
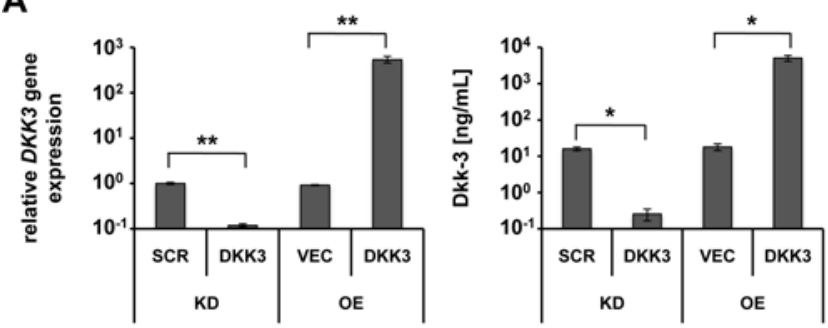

B

WST-1

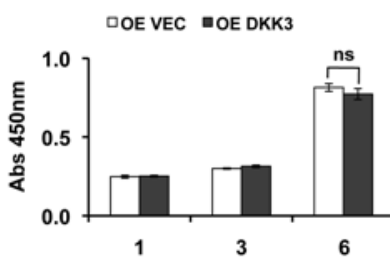

BrdU

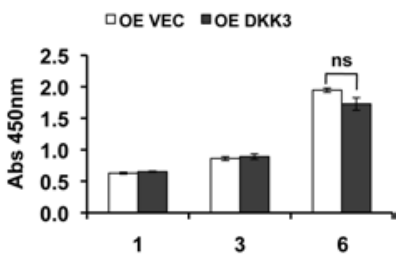

C
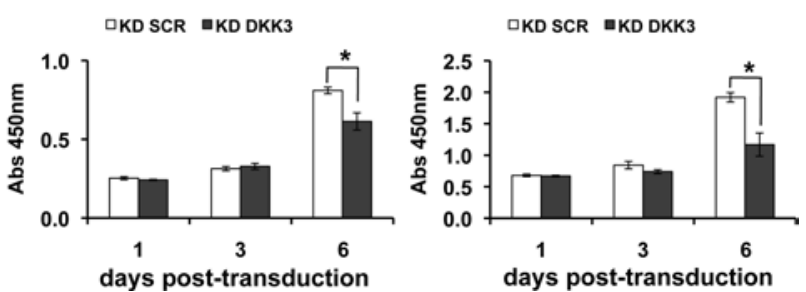

Figure 2. Influence of $D K K 3$ overexpression and knockdown on PANC-1 cellular proliferation. (A) Efficiency of $D K K 3$ overexpression and knockdown. $D K K 3$ mRNA levels and Dkk-3 protein levels determined in cell culture supernatants by IEMA of PANC-1 cells transduced with lentiviruses delivering shRNA targeting $D K K 3$ (KD DKK3), shRNA targeting scrambled control (KD SCR), full length $D K K 3$ (OE DKK3) or empty vector control (OE VEC), respectively. Knockdown lentiviruses (KD DKK3 and KD SCR) were applied at a MOI of 4, overexpression lentiviruses (OE DKK3 and OE VEC) at a MOI of 0.5. DKK3 gene expression levels were normalized using the housekeeping gene $H M B S$ and are shown relative to KD SCR PANC-1. (B) Lentiviral overexpression of $D K K 3$ (OE DKK3) did not affect proliferation of PANC-1 cells as determined by cell viability (WST-1) and DNA synthesis (BrdU incorporation) assays performed on day 1,3 or 6 post-transduction. (C) DKK3 knockdown (KD DKK3) significantly reduced proliferation of Dkk-3 expressing PANC-1 cells on day 6 post-transduction. Results represent mean values \pm SEM of three independent experiments. Statistical differences were calculated by paired Student's t-test and regarded significant when $\mathrm{P}<0.05\left({ }^{*} \mathrm{P}<0.05,{ }^{* *} \mathrm{P}<0.01\right)$.

malignancies (27), including PDAC (28). Thus, a panel of differentiation markers was analyzed to evaluate whether the observed reduced proliferation of KD DKK3 PANC-1 was associated with differentiation. First, activity of SA- $\beta$-Gal, a marker mainly correlated with senescence but also upregulated during differentiation (29), was assessed. In KD DKK3 PANC-1, an increased number of SA- $\beta$-Gal-positive cells compared with KD SCR cells was observed (Fig. 4A). Quantification of stained cells demonstrated that the increase was significant (Fig. 4B; KD SCR: $2.6 \%$ SA- $\beta$-Gal positive, KD DKK3: $12.2 \%, \mathrm{P}=0.002$ ). Moreover, mRNA levels of $G L B 1$, the enzyme responsible for SA- $\beta$-Gal staining, were significantly elevated in KD DKK3 PANC-1 (Fig. 4C; 5.0-fold, $\mathrm{P}=0.004$ ).

Subsequently, expression levels of marker genes of differentiated pancreatic epithelium were analyzed by qPCR in KD DKK3 and KD SCR PANC-1, respectively. Pancreatic amylase (AMY2A; 2.8-fold, $\mathrm{P}=0.008$ ), elastase 1 (CELA1; 7.8-fold, $\mathrm{P}=0.017)$, chymotrypsinogen $\mathrm{B} 1$ (CTRB1; 9.3-fold, $\mathrm{P}=0.013)$, 
A

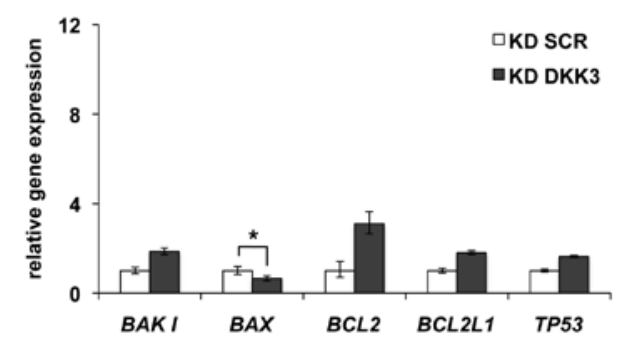

C

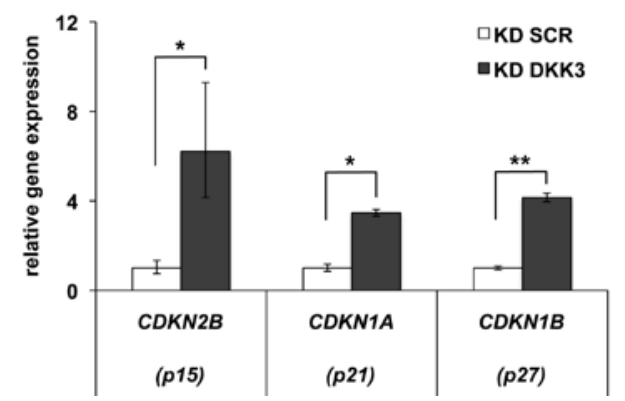

B

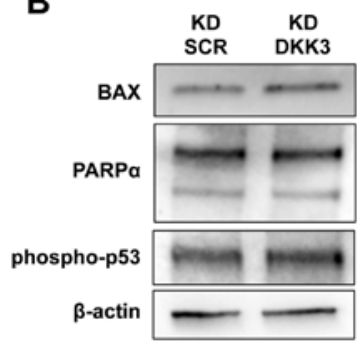

D

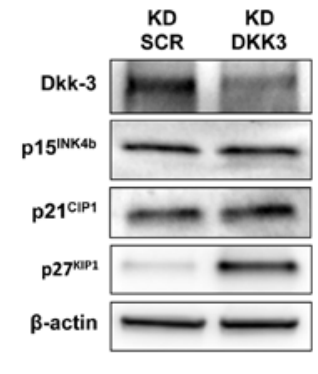

Figure 3. DKK3 knockdown induced cyclin-dependent kinase inhibitor expression in PANC-1 cells. (A) Expression of the apoptosis-related genes $B A K 1$, $B C L 2, B C L 2 L 1$ and TP53 was not affected by lentiviral-delivered shRNA targeting DKK3 (KD DKK3) compared with scrambled control (KD SCR) in PANC-1 cells. While BAX mRNA levels were slightly reduced by KD DKK3, protein levels were comparable to KD SCR (B). Protein levels of PARP $\alpha$ and phospho-p53 were not altered. (C) The cyclin-dependent kinase inhibitors $C D K N 2 B\left(\mathrm{p} 15^{\mathrm{INK} 4 \mathrm{~b}}\right), C D K N 1 A\left(\mathrm{p} 21^{\mathrm{CIPI}}\right)$ and $C D K N 1 B\left(\mathrm{p} 27^{\mathrm{KIPI}}\right)$ were significantly induced in KD DKK3 compared with KD SCR. (D) Induction of $\mathrm{p} 27^{\mathrm{KIP1}}$ was confirmed at protein levels by Western blot analysis while p15 $5^{\mathrm{INK} 4 \mathrm{~b}}$ and $\mathrm{p} 21^{\mathrm{CIP1}}$ protein levels were not affected. $\beta$-actin served as loading control. (A and C) Bars represent mean \pm SEM of four independent experiments.
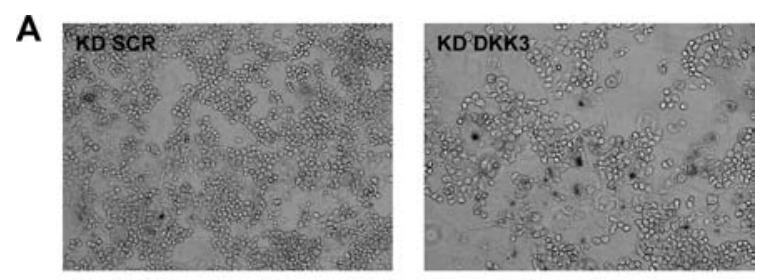

B
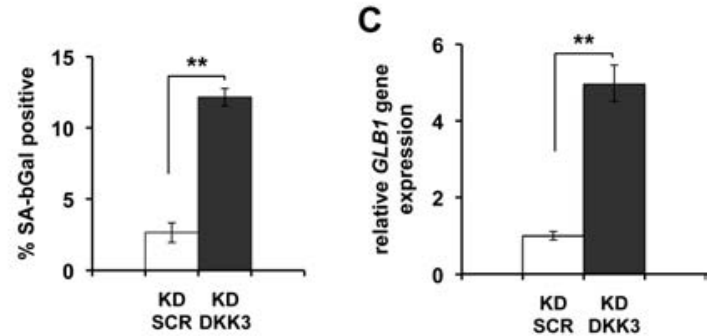

D

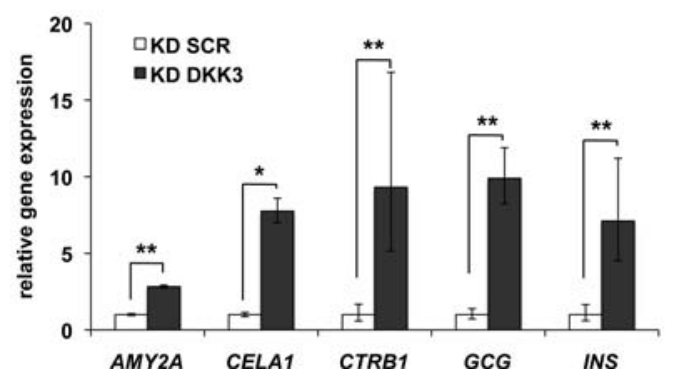

Figure 4. DKK3 knockdown induced expression of epithelial cell differentiation markers in PANC-1 cells. (A) DKK3 knockdown by lentiviral-delivered shRNA (KD DKK3) led to an increase of senescence-associated $\beta$-galactosidase (SA- $\beta$ gal)-positive PANC-1 cells on day 6 post-transduction. (B) Quantification of SA- $\beta$-gal stained-cells demonstrated that the observed increase was significant $(\mathrm{P}<0.01)$. (C) Gene expression of the SA- $\beta$-gal-active enzyme $G L B 1$ was significantly increased in KD DKK3 PANC-1, confirming the results of the SA- $\beta$-gal assay. (D) Markers for differentiated pancreatic epithelial cells were increased in KD DKK3 PANC-1. Data represent mean \pm SEM of three independent experiments. glucagon $(G C G$; 9.9-fold, $\mathrm{P}=0.007)$ and insulin (INS; 7.1-fold, $\mathrm{P}=0.003)$ were significantly induced in KD DKK3 cells (Fig. 4D).

To determine whether KD DKK3-induced differentiation of PANC-1 cells was similar to the formation of islet-like cell aggregates (ICAs), previously described (30), ICA formation was induced by switching PANC-1 cells to serum-free conditions in the presence of exendin-4. Induction of differentiation markers was comparable to KD DKK3 (Fig. 5A; AMY2A: 2.5-fold, $\mathrm{P}=0.049 ; C E L A 1: 2.7$-fold, $\mathrm{P}=0.12 ; C T R B 1: 5.7$-fold $\mathrm{P}=0.014 ; G C G$ : 14.6-fold, $\mathrm{P}=0.005$; INS: 5.7-fold, $\mathrm{P}=0.0003$ ). Moreover, expression of $G L B 1$ was significantly elevated (6.4-fold; $\mathrm{P}=0.032$ ) and exendin-4-differentiated cells showed high SA- $\beta$-Gal activity (Fig. 5B), confirming its suitability as differentiation marker. We also analyzed expression levels of the above-mentioned cell cycle-dependent kinase inhibitors, and while $C D K N 2 B$ mRNA levels were not significantly altered in exendin-4 induced differentiation (1.5-fold, $\mathrm{P}=0.188$ ), $C D K N 1 A$ (1.8-fold, $\mathrm{P}=0.014$ ) and $C D K N 1 B$ (2.9-fold, $\mathrm{P}=0.005$ ) levels were significantly increased (Fig. 5C). This expression pattern was confirmed at protein level (Fig. 5D). Interestingly, $D K K 3$ mRNA levels were slightly increased in differentiated PANC-1 cells (1.5-fold, $\mathrm{P}=0.024)$ and protein levels were comparable to control cells, indicating that while KD DKK3 induces differentiation markers in PANC-1, reduced Dkk-3 protein levels are not a prerequisite for differentiation.

\section{Discussion}

The PANC-1 cell line serves as a model for Dkk-3 expressing PDAC due to its expression DKK3 at mRNA levels (24). By confocal immunofluorescence, Western blot analysis and an IEMA based on our monoclonal antibody, Dkk-3 protein 
A

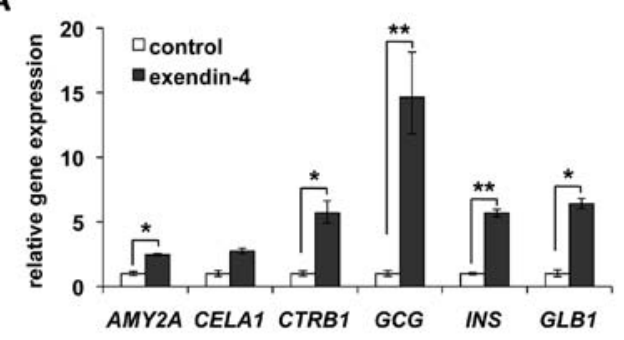

C

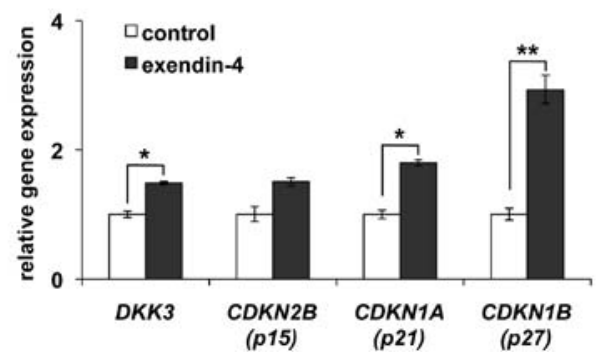

B

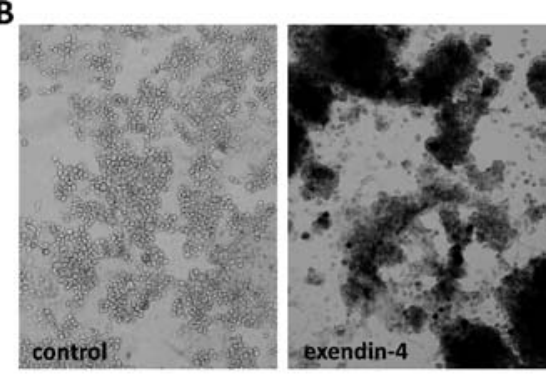

D

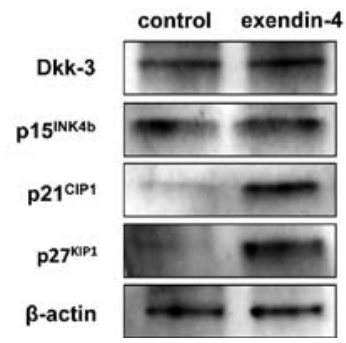

Figure 5. Marker profile of exendin-4 differentiated PANC-1 cells. (A) Pancreatic epithelial cell differentiation markers were increased in exendin-4-treated cells. (B) Elevated expression of $G L B 1$ was confirmed at enzyme activity levels using staining for senescence-associated $\beta$-galactosidase activity. Differentiation induced by exendin- 4 was accompanied by elevated $C D K N 1 A\left(\mathrm{p} 21^{\mathrm{CIPI}}\right)$ and $C D K N 1 B\left(\mathrm{p} 27^{\mathrm{KIPI}}\right)$ mRNA $(\mathrm{C})$ and protein $(\mathrm{D})$ levels, while $C D K N 2 B\left(\mathrm{p} 15^{\mathrm{INK} 4 \mathrm{~b}}\right)$ was not significantly changed compared with control-treated cells. DKK3 mRNA levels were found slightly increased in differentiated cells, whereas protein levels remained comparable with control. Data represent mean \pm SEM of three independent experiments.

expression was confirmed in PANC-1 cells and supernatants. Dkk-3 was heterogeneously distributed with a subpopulation of PANC-1 showing intense immunofluorescent staining. We previously reported similar results in the islets of Langerhans from normal adult human pancreas, where Dkk-3 was located only in a subpopulation of $\beta$ cells (17). The role of Dkk-3 in PANC-1 cells was investigated by generating lentiviral transduction systems to specifically overexpress or knockdown $D K K 3$, and those results verified the efficiency of the transduction system.

While OE DKK3 did not affect proliferation, KD DKK3 significantly reduced proliferation of PANC-1 cells, indicating that Dkk-3 was required to maintain the proliferative state of this cell line. KD DKK3 did not induce apoptosis, but led to a significant induction of cell cycle inhibitors and concomitantly increased gene expression levels of pancreatic epithelial cell differentiation markers, indicating that Dkk-3 is necessary for the maintenance of an undifferentiated state of PANC-1 cells. Our findings are surprising, since in other cell types, Dkk-3 has been shown to support differentiation. Depletion of Dkk-3 disrupted acinar morphogenesis of the prostate epithelial cell line RWPE-1 (31). Moreover, Dkk-3 supported capillary formation of peripheral blood-derived endothelial colony-forming cells (32). Taken together, Dkk-3 is either a key molecule stabilizing the differentiated as well as the undifferentiated state of cells, or has a distinct role in different cell types and cells of different origins.

In PANC-1 cells, Dkk-3 supports a highly proliferative, poorly differentiated state, both characteristics of progenitor or transit amplifying cells. As recently reported, Dkk-3 is strongly expressed at the base of colon crypts, a region known to contain proliferating epithelial precursor cells (33). Additionally, in skin hair follicles, Dkk-3 was preferentially expressed in the keratinocyte stem cell-containing bulge $(34,35)$. Dkk-3 has also been reported to represent a stemness gene in mesenchymal stem cells, where expression of Dkk-3 is reduced during differentiation (36). In a recent study, Dkk-3 overexpression was demonstrated in subsets of hepatoblastomas (11 of 14 cases) and hepatocellular carcinomas (14 of 72 cases), whereas the non-cancerous counterpart samples were negative for Dkk-3 (37). The authors suggested that Dkk-3 expression may distinguish a more immature or dedifferentiated subset of cancer. The question is whether the observed Dkk-3-positive PANC-1 fraction reflects a more progenitor-like subpopulation. If so, they provide an ideal model system to address further questions regarding the afore-mentioned hypothesis.

PDAC is a heterogeneous disease with several potential cellular origins $(38,39)$. In this context, the Dkk-3 overexpressing subset of PDACs might represent a subtype of PDACs with stem/progenitor cell origin.

At any rate, Dkk-3 expression distinguishes subsets of PDACs, which might require different treatments. Induction of ER stress by overexpression of Dkk-3 has been suggested as therapy for various cancers $(40,41)$. However, the requirement for Dkk-3 in PANC-1 cells to maintain their dedifferentiated phenotype indicates that this approach is inappropriate at least for Dkk-3 overexpressing PDACs. On the contrary, for this type of $\mathrm{PaCa}, \mathrm{Dkk}-3$ represents a potential target for functional neutralization in order to change the phenotype of the cancer cells to a more differentiated, and consequently less proliferative state.

In summary, KD DKK3 significantly reduced proliferation of PANC-1 and induced expression of epithelial cell differentiation markers. The results indicate that Dkk-3 maintains the tumor cells in a highly dedifferentiated state as a prerequisite for proliferation in the Dkk-3 overexpressing PDACs. Thus, Dkk-3 represents a potential target in this subset of PaCas. 


\section{Acknowledgements}

The authors are grateful to Martin Heitz and Anna Draxl for their excellent technical support. The manuscript was edited by M. K. Occhipinti-Bender.

\section{References}

1. Ferlay J, Parkin DM and Steliarova-Foucher E: Estimates of cancer incidence and mortality in Europe in 2008. Eur J Cancer 46: 765-781, 2010.

2. Jemal A, Siegel R, Ward E, Hao Y, Xu J and Thun MJ: Cancer statistics, 2009. CA Cancer J Clin 59: 225-249, 2009.

3. Cress RD, Yin D, Clarke L, Bold R and Holly EA: Survival among patients with adenocarcinoma of the pancreas: a population-based study (United States). Cancer Causes Control 17: 403-409, 2006

4. Krupnik VE, Sharp JD, Jiang C, et al: Functional and structural diversity of the human Dickkopf gene family. Gene 238: 301-313, 1999.

5. Niehrs C: Function and biological roles of the Dickkopf family of Wnt modulators. Oncogene 25: 7469-7481, 2006.

6. Wu W, Glinka A, Delius H and Niehrs C: Mutual antagonism between dickkopf 1 and dickkopf 2 regulates Wnt/beta-catenin signalling. Curr Biol 10: 1611-1614, 2000

7. Mao B, Wu W, Li Y, et al: LDL-receptor-related protein 6 is a receptor for Dickkopf proteins. Nature 411: 321-325, 2001.

8. Hsieh SY, Hsieh PS, Chiu CT and Chen WY: Dickkopf-3/REIC functions as a suppressor gene of tumor growth. Oncogene 23 : 9183-9189, 2004.

9. Tsuji T, Miyazaki M, Sakaguchi M, Inoue $\mathrm{Y}$ and Namba $\mathrm{M}$ A REIC gene shows down-regulation in human immortalized cells and human tumor-derived cell lines. Biochem Biophys Res Commun 268: 20-24, 2000.

10. Tsuji T, Nozaki I, Miyazaki M, et al: Antiproliferative activity of REIC/Dkk-3 and its significant down-regulation in non-smallcell lung carcinomas. Biochem Biophys Res Commun 289: 257-263, 2001

11. Nozaki I, Tsuji T, Iijima O, et al: Reduced expression of REIC/ Dkk-3 gene in non-small cell lung cancer. Int J Oncol 19: 117-121, 2001.

12. Kurose K, Sakaguchi M, Nasu Y, et al: Decreased expression of REIC/Dkk-3 in human renal clear cell carcinoma. J Urol 171: 1314-1318, 2004.

13. Kobayashi K, Ouchida M, Tsuji T, et al: Reduced expression of the REIC/Dkk-3 gene by promoter-hypermethylation in human tumor cells. Gene 282: 151-158, 2002.

14. Lodygin D, Epanchintsev A, Menssen A, Diebold J and Hermeking H: Functional epigenomics identifies genes frequently silenced in prostate cancer. Cancer Res 65: 4218-4227, 2005.

15. Zenzmaier C, Untergasser G, Hermann M, Dirnhofer S, Sampson N and Berger P: Dysregulation of Dkk-3 expression in benign and malignant prostatic tissue. Prostate 68: 540-547, 2008.

16. Fong D, Hermann M, Untergasser G, et al: Dkk-3 expression in the tumor endothelium: a novel prognostic marker of pancreatic adenocarcinomas. Cancer Sci 100: 1414-1420, 2009.

17. Hermann M, Pirkebner D, Draxl A, et al: Dickkopf-3 is expressed in a subset of adult human pancreatic beta cells. Histochem Cell Biol 127: 513-521, 2007.

18. Gray MJ, Wey JS, Belcheva A, et al: Neuropilin-1 suppresses tumorigenic properties in a human pancreatic adenocarcinoma cell line lacking neuropilin-1 coreceptors. Cancer Res 65: 3664-3670, 2005.

19. Glienke W, Maute L, Wicht J and Bergmann L: Wilms' tumour gene 1 (WT1) as a target in curcumin treatment of pancreatic cancer cells. Eur J Cancer 45: 874-880, 2009.

20. Cogoi S, Paramasivam M, Filichev V, Geci I, Pedersen EB and Xodo LE: Identification of a new G-quadruplex motif in the KRAS promoter and design of pyrene-modified G4-decoys with antiproliferative activity in pancreatic cancer cells. J Med Chem 52: 564-568, 2009.
21. Kondo J, Sato F, Kusumi T, et al: Claudin-1 expression is induced by tumor necrosis factor-alpha in human pancreatic cancer cells. Int J Mol Med 22: 645-649, 2008.

22. Hutzen B, Willis W, Jones S, et al: Dietary agent, benzyl isothiocyanate inhibits signal transducer and activator of transcription 3 phosphorylation and collaborates with sulforaphane in the growth suppression of PANC-1 cancer cells. Cancer Cell Int 9: 24, 2009.

23. Binker MG, Binker-Cosen AA, Richards D, Oliver B and CosenBinker LI: EGF promotes invasion by PANC-1 cells through Rac1/ROS-dependent secretion and activation of MMP-2. Biochem Biophys Res Commun 379: 445-450, 2009.

24. Takahashi N, Fukushima T, Yorita K, Tanaka H, Chijiiwa K and Kataoka H: Dickkopf-1 is overexpressed in human pancreatic ductal adenocarcinoma cells and is involved in invasive growth. Int J Cancer 126: 1611-1620, 2009.

25. Zenzmaier C, Marksteiner J, Kiefer A, Berger P and Humpel C: Dkk-3 is elevated in CSF and plasma of Alzheimer's disease patients. J Neurochem 110: 653-661, 2009.

26. Zenzmaier C, Sklepos L and Berger P: Increase of Dkk-3 blood plasma levels in the elderly. Exp Gerontol 43: 867-870, 2008.

27. Sgambato A, Cittadini A, Faraglia B and Weinstein IB: Multiple functions of $\mathrm{p} 27(\mathrm{Kip} 1)$ and its alterations in tumor cells: a review. J Cell Physiol 183: 18-27, 2000.

28. Karamitopoulou E, Zlobec I, Tornillo L, et al: Differential cell cycle and proliferation marker expression in ductal pancreatic adenocarcinoma and pancreatic intraepithelial neoplasia (PanIN). Pathology 42: 229-234, 2010

29. Untergasser G, Gander R, Rumpold H, Heinrich E, Plas E and Berger P: TGF-beta cytokines increase senescence-associated beta-galactosidase activity in human prostate basal cells by supporting differentiation processes, but not cellular senescence. Exp Gerontol 38: 1179-1188, 2003.

30. Hardikar AA, Marcus-Samuels B, Geras-Raaka E, Raaka BM and Gershengorn MC: Human pancreatic precursor cells secrete FGF2 to stimulate clustering into hormone-expressing islet-like cell aggregates. Proc Natl Acad Sci USA 100: 7117-7122, 2003.

31. Kawano Y, Kitaoka M, Hamada Y, Walker MM, Waxman J and Kypta RM: Regulation of prostate cell growth and morphogenesis by Dickkopf-3. Oncogene 25: 6528-6537, 2006.

32. Untergasser G, Steurer M,Zimmermann M, et al: The Dickkopfhomolog 3 is expressed in tumor endothelial cells and supports capillary formation. Int J Cancer 122: 1539-1547, 2008.

33. Byun T, Karimi M, Marsh JL, Milovanovic T, Lin F and Holcombe RF: Expression of secreted Wnt antagonists in gastrointestinal tissues: potential role in stem cell homeostasis. J Clin Pathol 58: 515-519, 2005.

34. Ohyama M, Terunuma A, Tock CL, et al: Characterization and isolation of stem cell-enriched human hair follicle bulge cells. J Clin Invest 116: 249-260, 2006.

35. Rittie L, Stoll SW, Kang S, Voorhees JJ and Fisher GJ: Hedgehog signaling maintains hair follicle stem cell phenotype in young and aged human skin. Aging Cell 8: 738-751, 2009.

36. Song L, Webb NE, Song Y and Tuan RS: Identification and functional analysis of candidate genes regulating mesenchymal stem cell self-renewal and multipotency. Stem Cells 24: 1707-1718, 2006.

37. Pei Y, Kano J, Iijima T, Morishita $Y$, Inadome $Y$ and Noguchi $M$ : Overexpression of Dickkopf 3 in hepatoblastomas and hepatocellular carcinomas. Virchows Arch 454: 639-646, 2009

38. Hernandez-Munoz I, Skoudy A, Real FX and Navarro P: Pancreatic ductal adenocarcinoma: cellular origin, signaling pathways and stroma contribution. Pancreatology 8: 462-469, 2008.

39. Stanger BZ and Dor Y: Dissecting the cellular origins of pancreatic cancer. Cell Cycle 5: 43-46, 2006.

40. Sakaguchi M, Kataoka K, Abarzua F, et al: Overexpression of REIC/Dkk-3 in normal fibroblasts suppresses tumor growth via induction of interleukin-7. J Biol Chem 284: 14236-14244, 2009.

41. Tanimoto R, Sakaguchi M, Abarzua F, et al: Down-regulation of BiP/GRP78 sensitizes resistant prostate cancer cells to genetherapeutic overexpression of REIC/Dkk-3. Int J Cancer 126: $1562-1569,2009$ 